\title{
Study on Fixed Salt Removal Effect by Fine Bubbles
}

\author{
Yuji MiKASA ${ }^{1}$, Naoya YAMAWAKI ${ }^{1}$, Hiroaki SHIDA ${ }^{2}$, Hayato OKUMURA ${ }^{3}$, Shigenori AKAMATSU ${ }^{3}$, \\ Yusuke NISHIUCHI ${ }^{3}$, and Takashi HATA ${ }^{3 *}$ \\ ${ }^{1}$ Advanced Course of Material Engineering, National Institute of Technology (KOSEN), Kochi College, 200-1 Monobe, Nankoku, \\ Kochi 783-8508, Japan \\ ${ }^{2}$ Ligaric Co., Ltd, 15-1- Shimizu, Suita, Osaka, 565-0805, Japan \\ ${ }^{3}$ Department of Social Design Engineering, National Institute of Technology (KOSEN), Kochi College, 200-1 Monobe, Nankoku, \\ Kochi 783-8508, Japan
}

\begin{abstract}
In recent years, it has become clear that $1 \mu \mathrm{m}$ or less sized fine air bubbles have different properties from those of $\mathrm{mm}$ or $\mathrm{cm}$ sized air bubbles. This study examined the effect of fine bubbles on the removal of salt fixed in the gap of aluminum plate as a verification of cleaning action by fine bubbles. As a result, it was confirmed that the removal effect of fixed salt is higher in fine bubble water than in control water, and that the degree of effect depends on the concentration of fine bubbles. It was also confirmed that less than $1 \mu \mathrm{m}$ sized ultrafine bubble water has a higher removal effect than the control water. As a mechanism of removing fixed salt, it is conceivable that peeling effect, like lifting up part of fixed salt, in which fine bubbles are dissolved by pressure change around the fixed salt to become supersaturated, causing further bubbling around there, and/or physical collision effect of microbubbles. In addition, it was confirmed that the removal effect of a system in which fine bubbles were regularly introduced was much higher than the one that does not introduce fine bubbles regularly. In addition, even when the gap between fixed salt and aluminum plate was narrowed, the superiority of fine bubble cleaning was confirmed.
\end{abstract}

\section{Introduction}

Recently, the International Organization for Standardization (ISO) defined fine bubbles as air bubbles of $100 \mu \mathrm{m}$ or less. By making the gas finer, fine bubbles have different specific properties from bubbles, which have size in $\mathrm{mm}$ and $\mathrm{cm}$ and are usually found in daily life. For example, the total surface area of multiple fine bubbles with the same volume as a single $\mathrm{cm}$-sized bubble is extremely large, thereby dramatically improving the chemical reaction, physical adsorption, and mass transport at the gas-liquid interface. Moreover, fine bubbles improve the dissolution efficiency of contained gas due to their huge total surface area. Fine bubbles have varied applications in agriculture and fisheries industry, environmental improvement, industrials, medical care, and food. Moreover, high environmental affinity and safety can be obtained by constructing these fine bubbles from air and water (Sivakumar and Pandit, 2002; Oshita, 2010; Terasaka, 2014a; Detlef, 2018).

Technological development related to the use of fine bubbles in cleaning is in its early stage. For example, fine bubbles have been introduced for cleaning in most toilets in expressway service areas in Japan and cleaning (removal) of sea salt particles flying in coastal structures. Although Ushida et al. (2011) reported that the surface tension of water-containing ultrafine bubbles (sized less than $1 \mu \mathrm{m}$ ) is about $10 \%$ lower than that of control water, there is a lack of sufficient academic verification. Therefore, in this research, the effect of fine bubbles on the removal of salt fixed to a gap of aluminum plate was examined to verify the cleaning action of fine bubbles.

\section{Experiment}

\subsection{Fine bubble generator}

BUVITAS (Ligaric Co. Ltd., Osaka, Japan) was used for generating fine bubbles. The generation mechanism is a gas-liquid shear system in which the gas shear field is obtained from the swirling of liquid. In addition, ion exchange water was used as water, and a hollow fiber filter was installed on the path to prevent mixing of impurities by introducing air.

\subsection{Measurements of particle size distribution and number density of fine bubbles}

ZetaView (MicrotracBEL Corp.), a particle tracking analysis (PTA) instrument, was used for measurement of particle size distribution and number density of ultrafine bubbles. PartAn SI (MicrotracBEL Corp.), an image

\footnotetext{
* Corresponding author: thata@ms.kochi-ct.ac.jp
} 
analyzer, was used for microbubble measurement. The ultrafine bubble measurements were made after floatation separation of micro bubbles.

\subsection{Preparation of a test plate and evaluation of removal (cleaning)}

The experimental system is shown in Figure 1. A 30-mm cylinder was attached to the center of a $100-\mathrm{mm}$ aluminum plate, a fixed layer of salt of about $2 \mathrm{~mm}$ was fabricated at a portion other than the surface of the plate attached to the cylinder, and a disk-shaped rotating plate was attached with a gap of $5 \mathrm{~mm}$. The test plate was immersed in $5 \mathrm{~L}$ fine bubble water diluted to a predetermined concentration to conduct agitation cleaning at a rotation speed of $40 \mathrm{rpm}$. To evaluate the removal of fixed salt, the concentration of salt removed from the test plate and dissolved in water was measured using electric conductivity. In other words, $w_{0} \mathrm{mg}$ weight of salt fixed to the test plate is measured with an electronic balance, and $w \mathrm{mg}$ weight of salt removed in the cleaning water is calculated from the electric conductivity to obtain the removal rate $\beta$ from the following Equation. In addition, the removed salt is assumed to be uniformly dissolved by the rotating machine and reflected in electric conductivity.

$$
\beta=\frac{w}{w_{0}} \times 100
$$

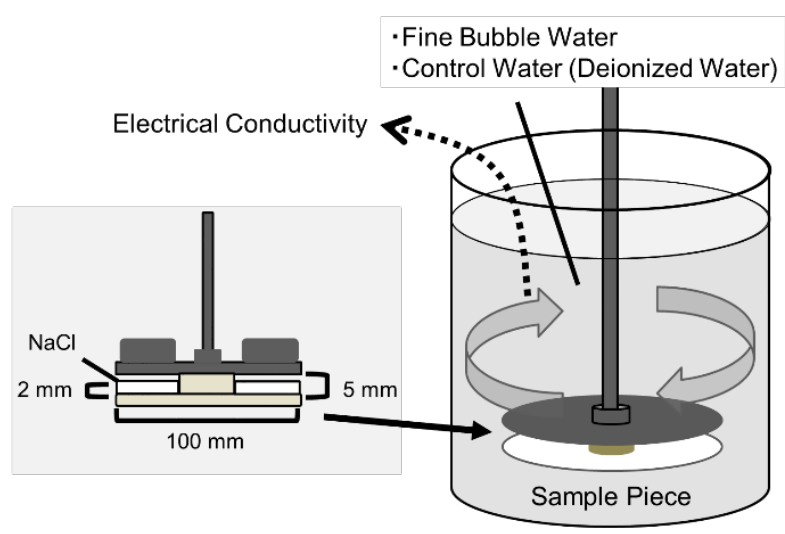

Figure 1. Schematic diagram of experiment.

\subsection{High-speed photography of fixed salt removal process}

For the fixed salt removal process, high-speed observation was performed using a photographing speed of 2000 fps and a shutter speed of 1/2000 sec using FASTCAM SA-Z made by PHOTRON LIMITED.

\section{Results and Discussion}

First, the particle size distribution of impurities contained in the ion-exchanged water, which is raw water, was measured and set as a baseline. After that, the particle size distribution of the water treated by BUVITAS was measured to determine the particle size distribution of fine bubbles. This resulted in two bubble groups of about $6.8 \times 10^{6}$ particles $\mathrm{mL}^{-1}$ (Figure 2 ) in the vicinity of $105 \mathrm{~nm}$ (ultrafine bubbles) and about $7.6 \times$ $10^{4}$ particles $\mathrm{mL}^{-1}$ (Figure 3 ) in the vicinity of $40 \mu \mathrm{m}$ (micro bubbles). Furthermore, in order to investigate the influence of number density of fine bubbles on the removal effect, fine bubbles were diluted to an arbitrary number density with ion-exchanged water before treatment. Their values are shown in Table 1. In addition, since the shrinkage behavior differs depending on the amount of dissolved gas, aeration treatment was

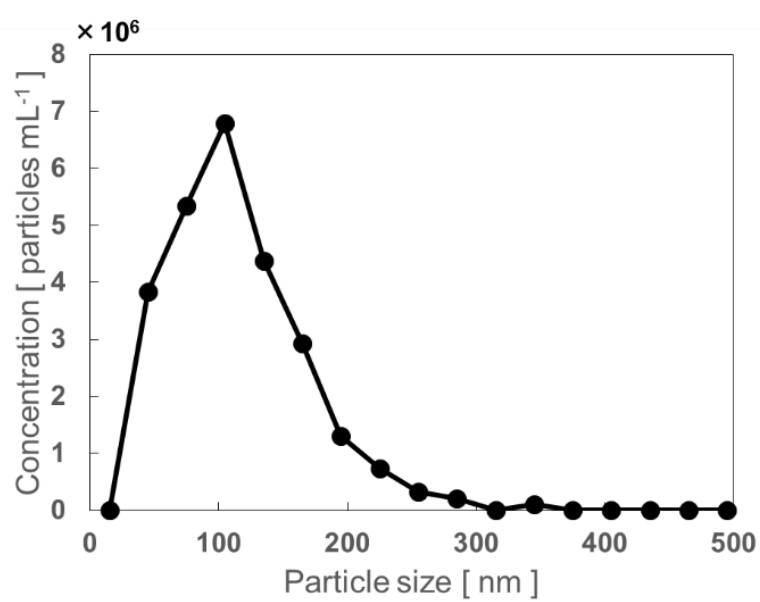

Figure 2. Particle size distribution of ultrafine bubbles.

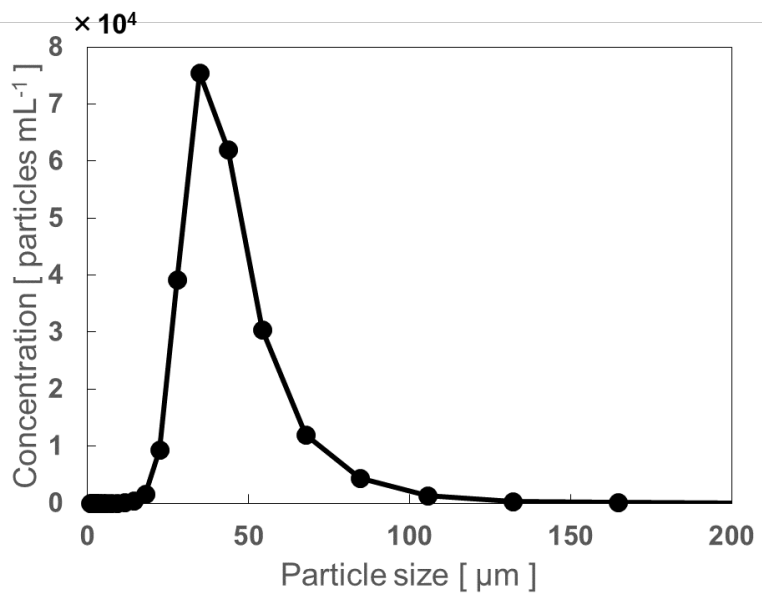

Figure 3. Particle size distribution of microbubbles.

Table 1. Number density of fine bubbles used in the experiment

\begin{tabular}{|c|c|c|}
\hline Sample & $\begin{array}{c}\text { Number density of } \\
\text { ultrafine bubble } \\
{\left[\text { particles } \mathrm{ml}^{-1} \text { ] }\right.}\end{array}$ & $\begin{array}{c}\text { Number density of } \\
\text { microbubble } \\
\text { [ particles } \mathrm{ml}^{-1} \text { ] }\end{array}$ \\
\hline \hline A & $2.5 \times 10^{7}$ & $17.8 \times 10^{4}$ \\
\hline B & $2.2 \times 10^{7}$ & $11.9 \times 10^{4}$ \\
\hline C & $1.2 \times 10^{7}$ & $5.9 \times 10^{4}$ \\
\hline D & $2.5 \times 10^{7}$ & - \\
UFB only & $0.3 \times 10^{7}$ & $1.0 \times 10^{4}$ \\
\hline E & \multicolumn{2}{|}{} \\
\hline
\end{tabular}




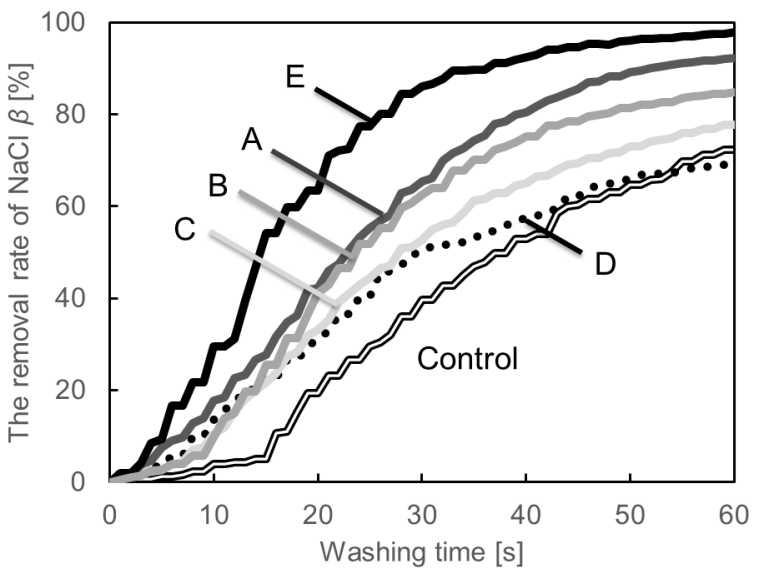

Figure 4. Removal behavior of fixed salt (for samples A to E, see Table 1)

conducted using an aeration pipe until the dissolved oxygen amount of the ion exchange water to be diluted is the same as that of fine bubble water. The shrinkage behavior of the fine bubbles is assumed to be constant in all the experimental systems.

Figure 4 shows the relationship between the cleaning time and fixed salt removal rate calculated from Equation (1). The experiment was repeated three times to show the average value. In the control water without fine bubbles, a rapid removal behavior was confirmed about 15 seconds after the gradual removal process immediately after the start of cleaning. On the other hand, in fine bubble systems (Sample A-C), the removal behavior was earlier than that of the control water immediately after the start of cleaning. It was also confirmed that its behavior depends on the number density of fine bubbles. Furthermore, in the system with only ultrafine bubbles (Sample D), although the removal rate eventually became the same as that of the control water, the removal behavior was observed to improve in the early stage. In order to verify this behavior, high speed camera photography near the test plate was performed 10 seconds after the start of cleaning in Sample D. The behavior is shown in Figure 5. While uniform dissolution behavior of fixed salt was observed in control water, peeling behavior of fixed salt was observed in the system with only ultrafine bubbles. In addition, the peeling behavior of fixed salt was also confirmed in the control water after about 15 seconds. Since this time is linked to the rapid removal behavior of the fixed salt observed in the control water of Figure 4, this behavior is considered to be related to the peeling of the fixed salt. Such a peeling behavior of fixed salt by ultrafine bubbles is also confirmed by Terasaka et al. (2014b) in their study of removing fixed salt on glass surface. Thus, the following jack-up theory is proposed. First, when ultrafine bubbles collide with fixed salt by water flow, they shift to a more stable state, i.e., a dissolved state in the liquid, due to pressure change. However, if this behavior occurs continuously, a high concentration dissolved gas region will be formed on the interface between the fixed salt and wall. When the dissolved gas concentration exceeds the degree of
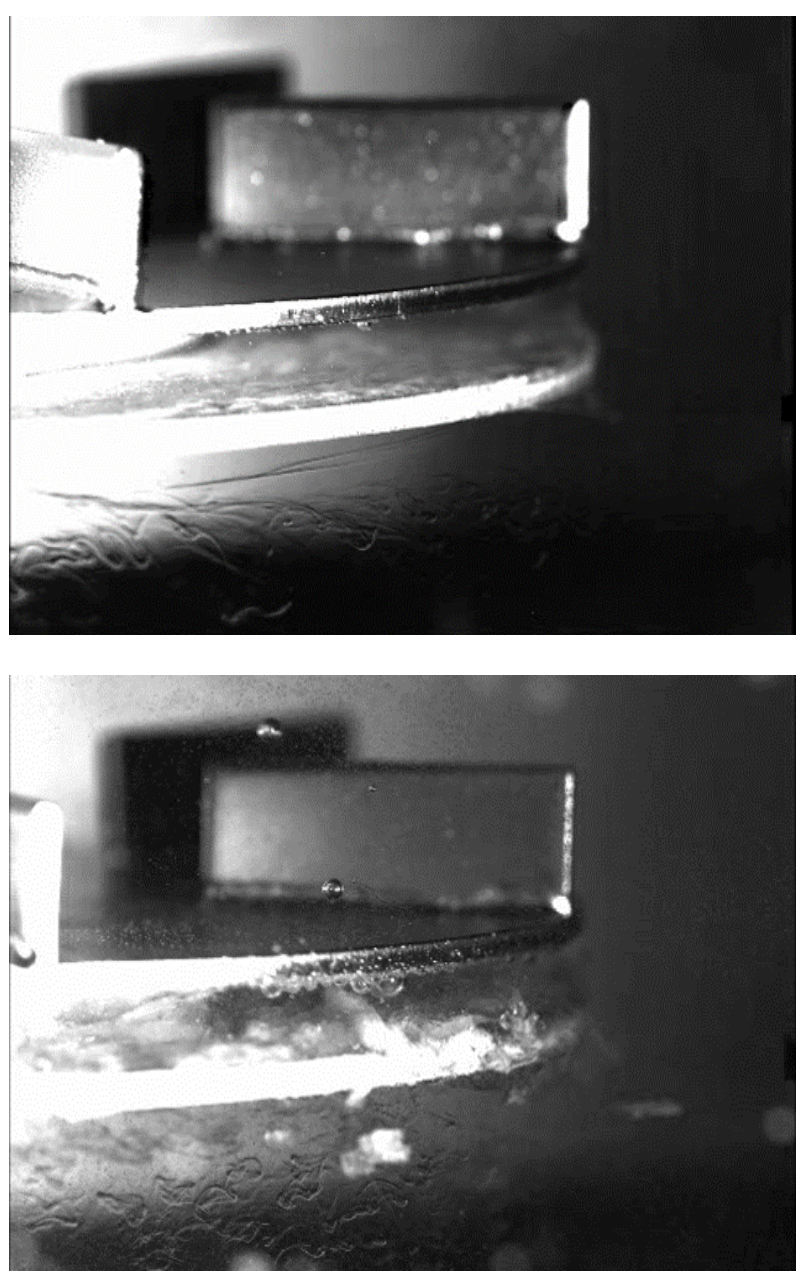

Figure 5. Removal behavior of fixed salt (above: control water, blow: ultrafine bubble water).

supersaturation, the gas phase will change to a more stable microbubble phase without returning to metastable ultrafine bubbles by forming a more stable gas phase than the dissolved state at the same portion. As a result, since microbubbles are much larger than the gap on the interface between the wall and fixed salt, part of the fixed salt is jacked up and inflow of water and/or collision of microbubbles will occur in the expanded gap. This will destroy the fixed salt with low ductility to progress the removal.

In this study, the phenomenon of jacking up was not confirmed in the control water adjusted to the same amount of dissolved oxygen as the system with only ultrafine bubbles. It is inferred that ultrafine bubbles act as a gas core causing a phase change to microbubbles, jacking up and peeling the fixed salt.

Furthermore, although the previous study by Terasaka et al. (2014b)is an experiment with a system of only ultrafine bubbles, the effect of entire fine bubbles containing micro bubbles as in this case is considered to have promoted the removal effect by the jack-up effect of ultrafine bubbles in addition to the physical collision action due to micro bubbles.

Next, when a quantitative evaluation was performed (Figure 4), the system with only ultrafine bubbles (Sample D) exhibited a higher removal rate after 30 seconds than that of the control water because of the 
fixed salt peeling effect immediately after the start of washing. However, after 60 seconds, the removal rate remained about $70 \%$, which is nearly the same rate as the control water. On the other hand, in the fine bubble water cleaning sample (Sample A-C) containing microbubbles, the removal effect was improved according to the number density of the fine bubbles; about $90 \%$ removal rate was obtained in Sample A with a high number density. In the experiments so far, fine bubble water was used at the start of the experiment, and fine bubbles were not added after starting the experiment. Therefore, a small fine bubble generator was connected to the cleaning tank of the experimental system to constantly supply fine bubbles. Here, a collision wall is provided so as to shield the water flow by introducing fine bubbles, ensuring that the structure of the system is not affected by the water flow. The number density of fine bubbles introduced at that time is shown as Sample $\mathrm{E}$ in Table 1. Although the number density was $10^{-1}$ (one order of magnitude) lower than Sample A, the removal behavior and rate were improved over Sample A as shown in Sample E in Figure 4 (about 98\% removal rate). This fact indicates that a part of fine bubbles disappears due to the jack-up phenomenon as described above in the cleaning using fine bubbles. This indicates the necessity of introducing constant fine bubbles to secure the cleaning effect.

Furthermore, by changing the gap on the aluminum plate in Figure 1 from $5 \mathrm{~mm}$ to $3 \mathrm{~mm}$ to fabricate a test plate, the same experiment was performed. Figure 6 shows the results using Sample B as the number density of fine bubbles. The experiment was repeated three times as in the previous experiment system to show the average value. Although there is no significant difference in the tendency of removal behavior including the removal time that occurs abruptly and the like, the final removal rate shows a decrease of about $10 \%$ in the control water due to the narrowing of the gap, while it was suppressed to about $5 \%$ in the fine bubble water. Moreover, this reduction suppression was intensified by the increase in the number density of fine bubbles, and the reduction in the removal rate because of the narrowing of the gap that was hardly observed when

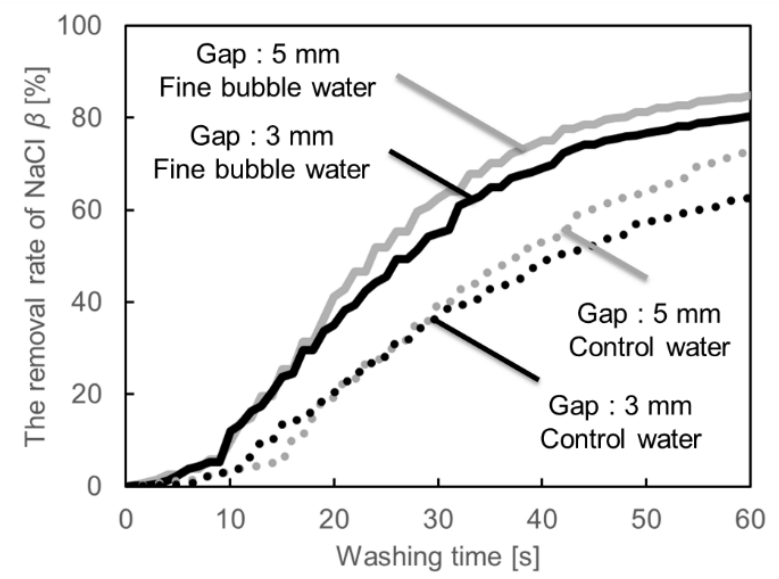

Figure 6. Relationship of a gap between fixed salt and aluminum plate affecting on the removal rate fixed of salt.
Sample A was used. The contribution of the surface tension reduction of ultrafine bubble water shown by Ushida et al. (2011) will be examined.

\section{Conclusion}

This study examined the effect of fine bubbles on the removal of salt fixed in a gap of aluminum plate. Iit was confirmed that the fine bubble water has a higher removal effect than the control water, and the degree of effect depends on the number density of fine bubbles. As a mechanism of removal, a jack-up effect caused by the dissolution of ultrafine bubbles and the transition to microbubbles, and/or the physical collision action of microbubbles may be considered. Moreover, further acceleration of cleaning effect was confirmed by introducing fine bubbles constantly. In addition, even when the gap between fixed salt and aluminum plate was narrowed, the superiority of the fine bubble washing was confirmed. From these results, usefulness of fine bubbles as a cleaning technology can be expected.

\section{References}

Detlef, L.; "Bubble Puzzles: From Fundamentals to Applications," Physical Review Fluids, 3, 110504 (2018)

Oshita, S.; "Present Situation of Micro- and NanoBubbles Technology," Food Processing and Ingredients, 45, 6-8 (2010)

Sivakumar M. and A. B. Pandit; "Wastewater Treatment: a Novel Energy Efficient Hydrodynamic Cavitational Technique," Ultrasonics Sonochemistry, 9, 123-131 (2002)

Terasaka, K.; "Trends and Challenges of Fine Bubble Technology," Chemical Engineering of Japan, 78, 580584 (2014a)

Terasaka, K., S. Fujioka, and M. Kawaragaki; "Applications of Fine Bubble Technology for IndustryCleaning with Ultrafine Bubble Water-," Proceedings of 2nd International Symposium on Multiscale Multiphase Process Engineering, pp. 148-153, Hamburg, Germany (2014b)

Ushida, A., T. Hasegawa, K. Amaki, T. Nakajima, N. Takahashi, and T. Narumi; "Investigation on Washing Effects for Nano-Bubble/Surfactant Mixtures in an Alternating Flow," Transactions of the Japan Society of Mechanical Engineers, 77, 1219-1228 (2011) 